\title{
Nuevos desafíos en la enseñanza y la pedagogía
}

Autora: Alegría de Lourdes Crespo Cordovez Universidad Internacional SEK, UISEK alegria.crespo@uisek.edu.ec Quito, Ecuador https://orcid.org/0000-0001-5716-9585

\section{Resumen}

El presente ensayo se plantea como objetivo poner en relevancia los principales desafíos de la educación en la actualidad, mismos que se relacionan tanto con la aparición de nuevas tecnologías que obligan al docente a replantear su actuación y participación en el escenario educativo. Se considera que el mayor de ellos supone el pasaje de una educación memorística, a una educación que tenga en cuenta las habilidades cognoscitivas, emocionales, creativas y morales de nuestros educandos, como lo sustentan los autores Bruner (1996); Fraca (2003); Temporetti (2006); y Vygotsky (2004). Al hacer participar a la psicología como un aliado estratégico en el proceso educativo, podemos comprender la importancia de alojar la subjetividad de niños, niñas y adolescentes, de manera que vivan y experimenten los procesos educativos desde un punto de vista participativo y comprometido con la construcción de nuevos paradigmas sociales y culturales.

Palabras clave: educación; psicología; enseñanza.

Código de clasificación internacional: 5802.04 - Niveles y temas de educación.

Cómo citar este ensayo:
Crespo, A. (2021). Nuevos desafíos en la enseñanza y la pedagogía. Revista Scientific, 6(22), 346-
$\begin{array}{ll}358, \quad \text { e-ISSN: 2542-2987. Recuperado de: } \text { https://doi.org/10.29394/Scientific.issn.2542- } \\ 2987.2021 .6 .22 .18 .346-358\end{array}$

Fecha de Recepción: 12-01-2021
Fecha de Aceptación: 10-06-2021
Fecha de Publicación: 05-11-2021 


\title{
New challenges in teaching and pedagogy
}

\begin{abstract}
The objective of this essay is to highlight the main challenges of education today, which are related both to the appearance of new technologies that force the teacher to rethink their performance and participation in the educational scenario. It is considered that the greatest of them supposes the passage from a memory education, to an education that takes into account the cognitive, emotional, creative and moral abilities of our students as supported by the authors Bruner (1996); Fraca (2003); Temporetti (2006); and Vygotsky (2004). By involving psychology as a strategic ally in the educational process, we can understand the importance of accommodating the subjectivity of children and adolescents, so that they live and experience educational processes from a participatory point of view and committed to the construction of new social and cultural paradigms.
\end{abstract}

Keywords: education; psychology; teaching. education.

International classification code: 5802.04 - Levels and subjects of

How to cite this essay:

Crespo, A. (2021). New challenges in teaching and pedagogy. Revista Scientific, 6(22), 346-358, e-ISSN: 2542-2987. Recovered from: https://doi.org/10.29394/Scientific.issn.2542-

2987.2021.6.22.18.346-358

Date Received: 12-01-2021
Date Acceptance: 10-06-2021
Date Publication: 05-11-2021 


\section{Introducción}

El análisis del proceso de enseñanza y del proceso de aprendizaje requiere pensamiento crítico y análisis exhaustivo de fenómenos que giran alrededor de él. Se debe contemplar la perspectiva pedagógica, social, antropológica y psicológica. Es importante contemplar la relación entre el profesor y el estudiante para comprender de qué manera el proceso cognitivo se desarrolla y así preparar al educando para servir a la sociedad con bases políticas.

Por tanto, es trascendental conocer y profundizar el amplio universo de la psicología y su relación con la educación para realmente comprender cómo ocurre el proceso de aprendizaje. Las teorías de aprendizaje han emergido, a partir del proceso cognitivo, en las cuales se han apoyado las estructuras psicopedagógicas de hoy mía. Paralelamente, se hará hincapié en la relación del educador y el educando como actores principales del proceso de aprendizaje. Para completar este análisis, se hará una revisión global de las responsabilidades que tenemos como educadores frente a una sociedad cada vez más inmediatista y adentrada en la tecnología.

El ser humano evoluciona permanentemente y, de la misma manera, lo hace la educación. Hoy en día se apunta a fortalecer el rol de mediador o facilitador para lograr ser un puente en el proceso de transmisión de conocimientos. Así mismo, el papel del estudiante es aquel de potenciar su aprendizaje a través del autoconocimiento, llegando a la metacognición con el fin de optimizar las oportunidades de aprendizaje. Es importante que tanto educador como educando, hagan real conciencia de lo trascendental de asumir su rol con responsabilidad para lograr resultados óptimos.

La búsqueda del hombre para conocer cómo se da el proceso de aprendizaje y de cognición en la historia es mucho más antigua que la psicología estructurada como tal. Para Hardy y Harris (1998a): los filósofos griegos ya indagaban cómo se desarrolla la manera de aprender del ser 
humano. Las ideas de los filósofos han influido notablemente en cómo los psicólogos estudian el proceso de aprendizaje, así como las teorías existentes en torno a este proceso.

El presente ensayo se plantea como objetivo trazar las nuevas formas de actuación y participación del docente en el escenario educativo, de manera que los fundamentos de su práctica se encuentren adaptados a las nuevas realidades técnicas, curriculares y psicológicas de los educandos.

\section{Desarrollo}

La psicología, en sus inicios, constituía parte de la filosofía y, por esta razón, contempla dos problemas fundamentales: el primero, cómo aprendemos y el segundo, el problema en torno a cómo aprenden las otras mentes. Se manifiesta la epistemología como una subdisciplina de la filosofía, la misma que trata de la génesis del conocimiento humano.

Tomando este fundamento como punto de partida, cabe manifestar que la teoría más antigua del conocimiento es la "Teoría de la Copia" que fue propuesta por Demócrito citado por Cañas (2012): quien manifiesta que el ser humano percibe un objeto y crea una copia en su mente. Según Hardy y Harris (1998b): se encuentra con el problema del escepticismo puesto que cada interpretación realizada por cada mente humana puede diferir significativamente de la realidad.

Frente a este problema de escepticismo surgen tres reacciones mencionadas por Hardy y Harris (1998c), Realismo: elimina la copia con el fin de restaurar el mundo real lo más cercano posible a su esencia. Lo que pretende es que conozcamos los objetos directamente de la realidad; Idealismo: las ideas, y no los objetos, son las que conforman nuestro conocimiento; y Pragmatismo: contradice al escepticismo argumentando que, si estamos equivocados de una cosa, no estamos equivocados del todo.

La psicología dedicada al aprendizaje contempla a la biología como un 
gran apoyo por dos razones: por un lado, el aprendizaje representa un cambio

o transformación en el sistema nervioso de la persona. Por otro lado, para Hardy y Harris (1998d): surge el enunciado del nativismo que cree que el aprendizaje es innato. En este sentido, Dewey (1977a), menciona que:

Toda educación procede por la participación del individuo en la conciencia social de la raza. Este proceso comienza inconscientemente casi desde el nacimiento, y está continuamente formando las capacidades del individuo, saturando su conciencia, formando sus hábitos, educando sus ideas y despertando sus sentimientos y emociones (pág. 1).

Tomando en cuenta este enunciado de Dewey (1977b): la educación debe ser la fuente de potenciación del aprendizaje a través de las herramientas, actividades e insumos adecuados. La escuela es una institución meramente social y se basa en ser la representante de la vida comunitaria replicando los elementos más importantes de la vida para preparar al estudiante con miras a ser un ente que se inserte en la sociedad de manera asertiva. El objetivo real de las instituciones educativas radica en pulir, perfeccionar y potenciar a los estudiantes para que calcen socialmente y signifiquen un aporte.

Han emergido teorías del aprendizaje que sustentan el proceso de adquisición de conocimiento y procuran aclararlo. El Conductismo se considera como la única teoría psicológica que contempla las características para ser una teoría de aprendizaje, señalando a Temporetti (2009): está construida en el marco conceptual y de metodología de la tradición del positivismo y del empirismo.

Al emplear el método científico, el conductismo de Bunge y Ardila (2002): se posiciona sobre hechos y objetividad para examinar el comportamiento de los animales siendo ésta un resultado de la estimulación externa. La escuela del conductismo surge antes de la Primera Guerra Mundial en los Estados Unidos. Tuvo mucha influencia sobre la psicología, sin 
embargo, perdió fuerza. El conductismo nace contradiciendo el mentalismo tradicional y rechazando el crecimiento de las facultades de la mente y tomando en cuenta la introspección como un elemento primordial.

De acuerdo con Skinner (1964); y Watson (1913), citados por Rodríguez (2010): el conductismo clásico, adapta al positivismo la metafísica y la gnoseología. Hay objetos, sucesos o apariencias que se presentan ante un observador ante la metafísica positivista. Anteriormente, no se asociaba la ciencia con la pedagogía, los conductistas consideraban que gracias a su corriente y su escuela se acercaba la pedagogía a la ciencia. Mencionando a Temporetti (2006a): la pedagogía distaba de ser científica, pero el conductismo la podía acercar a ser una ciencia, conforme a los conductistas. Los psicólogos conductistas consideraban que, si ellos comprenden las leyes del aprendizaje en el laboratorio, el paso a seguir era que los profesores las interioricen y apliquen en sus clases. Solamente de esta manera, la pedagogía se convertiría en científica. Muchos casos fueron exitosos, otros no.

Se ha dado un cambio de dirección en la teoría psicológica científica en los últimos años. Posteriormente a la psicología basada en el conductismo, se da un nuevo paradigma y es la psicología basada en el cognitivismo la cual se basa en cómo la información es procesada. El mismo se basa en una comparación entre el funcionamiento de la mente humana y los computadores. De esta manera, se contempla cambios en la perspectiva de procesos mentales como por ejemplo la memoria y la atención. Para Pozo (1997): las posiciones conductuales siguen primando en la psicología del aprendizaje. Justamente las teorías del aprendizaje emergen de las teorías cognitivas desde sus diversos enfoques.

Señalando a Temporetti (2006b): muchos proyectos interesantes de psicología quisieron llegar a tan altos niveles. Por eso podemos notar que, por el contario, la Psicología Pragmática contiene en su desarrollo una Teoría del Aprendizaje, a pesar de que intentan encaminar al profesor para alcanzar los 
objetivos de aprendizaje de sus estudiantes.

Es complejo preparar a una nueva generación en una época desconcertante con respecto a la educación. Esto lo manifiesta Bruner (1996a): padre de la Psicología Cultural. El autor hace hincapié en que la sociedad, en plena evolución y transformación, exige preparar a estudiantes para que sean capaces de enfrentarla. Además, si hacemos un análisis sobre la transformación educativa durante la pandemia del COVID-19, nos encontramos frente a un desafío aún más grande en el cual la tecnología se convierte en protagonista.

En ese marco, Bruner (1996b): hace un análisis interesante sobre el significado de los conceptos sociales, manifestando que le pertenecen a aquel que les da el significado, por lo cual pueden variar de una persona a otra. Así, el profesor o educador tiene la responsabilidad de ser facilitador y mediador.

De esta manera, el aprendizaje es un proceso subjetivo, la realidad presenta distintos conceptos dependiendo de la persona que está actuando como el estudiante o interiorizando el aprendizaje. El educador es, entonces, mediador y facilitador de aprendizaje. En esa misma línea, Bruner (1996c): menciona que el lenguaje que adoptan los profesores entre sí, demuestra más incertidumbre que aquel lenguaje que utiliza el profesor con sus estudiantes debido a que debe transmitir con seguridad y estabilidad los conocimientos.

Es importante analizar la posición del educador frente al educando. El giro y el enfoque que le da el docente a la información son elementales para un proceso de aprendizaje exitoso. Parafraseando a Bruner (1996d): es importante que el profesor vea en la educación una oportunidad en la cual el estudiante es protagonista y se apropia del aprendizaje. En referencia al tema, Fernández y Atucha (2011): llaman a este fenómeno "elaboración de la cultura" (pág. 2); lo cual pretende convertir algo cotidiano en algo extraordinario para ganar la atención del estudiante.

Urge remontarse a la concepción de Piaget (1972), citado por Bruner, 
(1996e): sobre el niño, ya que lo veía como un aprendiz autónomo y apropiado de su aprendizaje, postulado al cual apoya en varios de sus escritos. Con esta investigación, se deduce que el niño se apropia de su aprendizaje y lo convierte en un modelo para sí mismo. Esta interiorización o apropiación está enmarcada en un contexto sociocultural que logra que el niño adapte la realidad y el aprendizaje en sus propios términos.

Sin duda, cabe apelar al legado de Vygotsky (2004a): para apoyar este análisis. El psicólogo identifica la relación exacta entre pensamiento, socialización y lenguaje. Una de sus ideas más importantes es que el adulto constituye un ejemplo para el niño con el fin de que lo imite $y$, a través del diálogo, va dándole pautas para que el aprendiz llegue cada vez más arriba y tenga interiorizado el proceso para, posteriormente, realizarlo solo.

Para Vygotsky (2004b): la actividad y la mediación son dos nociones básicas para comprender y fundamentar el modelo de aprendizaje. Por un lado, el concepto de actividad se refiere al motor del ser humano, mientras que la mediación se asemeja al concepto de adaptación. Así, para Fraca (2003a): la actividad se convierte, entonces, en un proceso de transformación del entorno mediante la mediación.

Para que el proceso de mediación resulte óptimo, la cultura es un pilar estructural, pues contempla elementos como el lenguaje. Cabe recalcar que todo proceso de lenguaje inicia siendo un proceso interpersonal y se va convirtiendo e instaurando en un proceso intrapersonal. Como dice Fraca (2003b): los significados son reconstruidos permanentemente pues ya son parte de la historia, la cultura y las tradiciones populares.

El educador, en conformidad con Fraca (2003c): debe ser sumamente perceptivo e intuitivo para identificar hasta dónde puede llegar el estudiante y, de esta manera, utilizar actividades y herramientas apropiadas para potenciar su aprendizaje. Por lo que, el papel del educador adquiere un concepto motivador fundamental que trasciende su conocimiento sobre la materia y 
reposa sobre sus estrategias para potenciar y enriquecer las capacidades del educando. El diseño curricular y microcurricular que emplee es trascendental para llegar a niveles altos con su aprendiz.

\section{Conclusiones}

La manera de estructurar la realidad a través de una expresión humana se da a través del lenguaje. De esta manera, se delimita la interacción entre educador y educando al estructurar la interpretación de la realidad.

Se hace énfasis en las teorías de aprendizaje, mismas que quieren asociar a la psicología con la educación. Si bien la teoría otorga información valiosa, puede interrumpir el proceso fluido y espontáneo de la enseñanza y del aprendizaje.

El aprendizaje "mimético" en el cual el profesor actúa y el alumno imita, de esta manera la imitación es motor y el estudiante debe estar muy apegado a la información brindada y no salirse del carril para seguir la estructura de la enseñanza. De esta manera, se fomenta el aprendizaje automatizado, mecánico y sin pensamiento crítico que no posiciona al estudiante como dueño de su proceso de aprendizaje impidiendo que haya verdadera retroalimentación y potenciación del aprendizaje. Lo que se pretende es que el alumno sea analítico, reflexivo y este tipo de aprendizaje limita el pensamiento y la adquisición de conocimiento a un proceso mecánico.

El maestro debe procurar cumplir el papel de facilitador o mediador para que el alumno sea protagonista y sea generador de ideas y opiniones demostrando su comprensión. Es sumamente interesante encontrar que el contraste entre el modo mimético y de transformación, se basa en habilidades básicas y creatividad.

Lo interesante es que en la enseñanza mimética que fomenta las habilidades básicas, el aprendizaje estará enfocado en destrezas básicas como identificar, comprender, comparar, para que, con mayor fortaleza y 
posteriormente, se construya con creatividad a partir de esa base. El educador que se inclina y fomenta la creatividad, ve en sus estudiantes generadores de nuevas ideas, de contenido, de productos que pueden llevar al pensamiento a su máximo potencial y pasar de ser pensamiento de orden inferior a pensamiento de orden superior.

El educador es un agente predominante en el proceso de aprendizaje efectivo, y debe ser sobre alguien atento, sensible, que sepa escuchar y observar los procesamientos individuales y grupales.

Históricamente, las funciones de un profesor estaban muy delimitadas y definidas; su papel se reducía a transmitir la información que estaba ligada a las ciencias que enseñaba, es decir, era un "instructor". A medida que la sociedad evoluciona, también lo hace la función del profesor y pasa a ser de instructor a educador, su función también pasa a ser más amplia. En las primeras décadas del siglo $\mathrm{XX}$, el maestro era como el hombre del Renacimiento, al cual se exigía que inserte al niño desde lo físico a lo intelectual, en sus diferentes aspectos científico y humanístico.

Esta es una breve descripción del rol del educador. Cabe entonces analizar más profundamente el rol del educador en la actualidad. Nos vemos sumergidos en una época llena de estímulos, en la cultura del consumismo, de lo descartable, de la satisfacción inmediata y de la condescendencia.

Se destaca vehementemente el rol del profesor respetado como autoridad, como ejemplo a seguir por sus conocimientos, por su experiencia, por su trayectoria. La crítica que emerge bajo mi opinión es el fomento a una educación irreflexiva y automática, sin embargo, rescato metodologías que nos ayudaban a memorizar y a asumir nuestro rol como educandos con respecto al educador.

Sin embargo, esta actitud fomenta a la interiorización del conocimiento y a que el educando se adueñe de dicho conocimiento y se sienta partícipe. Como educadores, nos encontramos ante un desafío intelectual y moral, muy 
rico, que nos impulsa a prepararnos permanentemente para trascender en la memoria de nuestros estudiantes como entes generadores de curiosidad y guías a las cuales volverán en distintas situaciones de su vida. El desafío se torna aún mayor debido a la tecnología que nos permite transportarnos en milésimas de segundos a cualquier lugar del mundo y a cualquier tipo de información.

Por lo tanto, debemos ser educadores atrevidos, seguros, capacitados, perspicaces que utilicen la información brindada hasta la actualidad por grandes pensadores, nuestra propia experiencia como educandos y la corriente actual de satisfacer las necesidades cognitivas, físicas, emocionales, creativas y morales que tenemos en bandeja de plata en cada uno de nuestros educandos.

\section{Referencias}

Bunge, M., \& Ardila, R. (2002). Filosofía de la Psicología. ISBN: 968-232325-8. Barcelona, España: Siglo Veintiuno Editores, S.A.

Bruner, J. (1996a,b,c,d,e,f). Realidad mental y mundos posibles: Los actos de la imaginación que dan sentido a la experiencia. Tercera reimpresión, ISBN: 84-7432-313-4. Barcelona, España: Editorial Gedisa, S.A.

Cañas, R. (2012). La estructura de la materia en los filósofos pluralistas pre-socráticos. InterSedes: Revista de las Sedes Regionales, 13(25), 143-168, e-ISSN: 2215-2458. Recuperado de:

https://www.redalyc.org/articulo.oa?id=66623936008

Dewey, J. (1977a,b). Mi credo pedagógico: Teoría de la educación y sociedad. Buenos Aires, Argentina: Centro Editor de América Latina.

Fernández, C., \& Atucha, H. (2011). La cultura como proceso de producción y apropiación espiritual. Contribuciones a las Ciencias Sociales, (12), 1-5, e-ISSN: 1988-7833. España: Universidad de 
Málaga; Servicios Académicos Intercontinentales, S.L.

Fraca, L. (2003a,b,c). Pedagogía integradora en el aula. Teoría, práctica y evaluación de estrategias de adquisición de competencias cognitivas y lingüísticas para el empleo efectivo de la lengua materna oral y escrita. Caracas, Venezuela: Libros El Nacional.

Hardy, T., \& Harris, R. (1998a,b,c,d). Aprendizaje y Cognición. ISBN: 848322-016-4. Madrid, España: Prentice Hall.

Pozo, J. (1997). Teorías cognitivas del aprendizaje. ISBN: 978-84-7112581-1. Madrid, España: Ediciones Morata, S.L.

Rodríguez, E. (2010). Bases filosóficas del análisis de la conducta. Perspectivas Psicológicas, 6-7, e-ISSN: 1992-5131. República Dominicana: Instituto de Psicología de la Universidad Autónoma de Santo Domingo (Facultad de Humanidades).

Temporetti, F. (2006a,b). Teoría psicológica y prácticas educativas: hacia una psicología más interpretativa en el proceso de enseñar y aprender. Rosario, provincia de Santa Fe, Argentina: Universidad Nacional de Rosario.

Temporetti, F. (2009). Teorías del aprendizaje y práctica docentes con niños. Seminario. Rosario, provincia de Santa Fe, Argentina: Facultad de Humanidades y Artes de la Universidad Nacional de Rosario.

Vygotsky, L. (2004a,b). Imagination and Creativity in Childhood. Journal of Russian and East European Psychology, 42(1), 7-97, e-ISSN: 10610405. Recovered from:

https://doi.org/10.1080/10610405.2004.11059210 


\section{Ensayo Original / Original Essay}

\section{Alegría de Lourdes Crespo Cordovez \\ e-mail: alegria.crespo@uisek.edu.ec}

Nacida en Quito, Ecuador, el 27 de septiembre del año

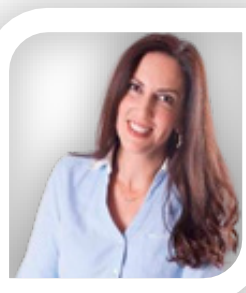
1975. Doctora en Ciencias de la Educación por la Universidad Nacional de Rosario (UNR), Argentina; Máster en Educación por la Universidad San Francisco de Quito (USFQ), Ecuador; actual Directora de Educación Online-Digital School de la Universidad Internacional SEK (UISEK); Columnista de revistas y publicaciones de opinión en temas de educación, política y sociedad a nivel nacional; Consultora en temas de calidad de la educación y asesoramiento comunicacional y pedagógico.

El contenido de este manuscrito se difunde bajo una Licencia de Creative Commons ReconocimientoNoComercial-Compartirlgual 4.0 Internacional 